\title{
Experimental analysis of out-of-plane Lorentz force actuated magnetic field sensor
}

\author{
Muhammad U. Mian ${ }^{1 a)}$, John O. Dennis ${ }^{2}$, Mohd H. M. Khir ${ }^{1}$, \\ Mawahib G. A. Ahmed ${ }^{1}$, Almur A. S. Rabih ${ }^{1}$, \\ and Tong Boon Tang ${ }^{1}$ \\ ${ }^{1}$ Department of Electrical and Electronic Engineering, Universiti Teknologi \\ PETRONAS, 32610 Bandar Seri Iskandar, Perak, Malaysia \\ ${ }^{2}$ Department of Fundamental and Applied Sciences, Universiti Teknologi \\ PETRONAS, 32610 Bandar Seri Iskandar, Perak, Malaysia
}

a)umermian@gmail.com

\begin{abstract}
In this article, we present a simple MEMS magnetic sensor based on Lorentz Force principle. In this work, the sensor is designed, fabricated and characterized capacitively using a standard capacitance to voltage MS3110 circuit. The sensor is fabricated based on double thickness PolyMUMP surface micromachining process. In this process, the two Poly layers are combined to increase the thickness of the sensor beams and central shuttle. In response to an input excitation current an out of plane motion due to Lorentz force occurs which is detected by the change in capacitance between the moving and static plate. The experimentally detected resonant frequency of the sensor is $5.1 \mathrm{kHz}$. The experimental sensitivity achieved by the sensor at atmospheric condition is $5.59 \mathrm{~V} / \mathrm{T}$ for the input current of $30 \mathrm{~mA}$ with a damping ratio of 0.010 .
\end{abstract}

Keywords: MEMS, PolyMUMP, Lorentz force, capacitive, spring softening, MS3110

Classification: Micro- or nano-electromechanical systems

\section{References}

[1] J. Lenz, et al.: "Magnetic sensors and their applications," IEEE Sens. J. 6 (2006) 631 (DOI: 10.1109/JSEN.2006.874493).

[2] A. L. Herrera-May, et al.: "Mechanical behavior of a novel resonant microstructure for magnetic applications considering the squeeze-film damping," Microsyst. Technol. 15 (2009) 259 (DOI: 10.1007/s00542-008-0658-4).

[3] H. Emmerich and M. Schofthaler: "Magnetic field measurements with a novel surface micromachined magnetic-field sensor," IEEE Trans. Electron Devices 47 (2000) 972 (DOI: 10.1109/16.841228).

[4] B. Bahreyni and C. Shafai: "A resonant micromachined magnetic field sensor," IEEE Sens. J. 7 (2007) 1326 (DOI: 10.1109/JSEN.2007.902945). 
(2016) 88 (DOI: 10.3390/mi7050088).

[6] M. Li, et al.: "Three-axis Lorentz-force magnetic sensor for electronic compass applications," J. Microelectromech. Syst. 21 (2012) 1002 (DOI: 10.1109/ JMEMS.2012.2196493).

[7] J. A. Tapia, et al.: "Sensing magnetic flux density of artificial neurons with a MEMS device,” Biomed. Microdevices 13 (2011) 303 (DOI: 10.1007/s10544010-9494-2).

[8] J. Yunas, et al:: "Spin-on-glass (SOG) based insulator of stack coupled microcoils for MEMS sensors and actuators application," Sains Malays. 43 (2014) 289.

[9] C.-I. Chang, et al.: "Development of CMOS-MEMS in-plane magnetic coils for application as a three-axis resonant magnetic sensor," J. Micromech. Microeng. 24 (2014) 035016 (DOI: 10.1088/0960-1317/24/3/035016).

[10] X. Lv, et al.: "A novel MEMS electromagnetic actuator with large displacement," Sens. Actuators A Phys. 221 (2015) 22 (DOI: 10.1016/j.sna.2014.10. 028).

[11] V. Beroulle, et al.: "Monolithic piezoresistive CMOS magnetic field sensors," Sens. Actuators A Phys. 103 (2003) 23 (DOI: 10.1016/S0924-4247(02)003175).

[12] A. L. Herrera-May, et al.: "A resonant magnetic field microsensor with high quality factor at atmospheric pressure,” J. Micromech. Microeng. 19 (2009) 015016 (DOI: 10.1088/0960-1317/19/1/015016).

[13] P. Gkotsis, et al.: "Mechanical characterization and modelling of Lorentz force based MEMS magnetic field sensors," Solid-State Electron. 112 (2015) 68 (DOI: 10.1016/j.sse.2015.02.004).

[14] C.-I. Chang, et al.: "Development of CMOS-MEMS in-plane magnetic coils for application as a three-axis resonant magnetic sensor," J. Micromech. Microeng. 24 (2014) 035016 (DOI: 10.1088/0960-1317/24/3/035016).

[15] D. Ren, et al.: "Design and analyses of a MEMS based resonant magnetometer," Sensors 9 (2009) 6951 (DOI: 10.3390/s90906951).

[16] C.-H. Hsieh, et al.: "Fabrication and characterization of CMOS-MEMS magnetic microsensors," Sensors 13 (2013) 14728 (DOI: 10.3390/ s131114728).

[17] B. Park, et al.: "Lorentz force based resonant MEMS magnetic-field sensor with optical readout," Sens. Actuators A Phys. 241 (2016) 12 (DOI: 10.1016/ j.sna.2016.01.032).

[18] J. E.-Y. Lee, et al.: "An axial strain modulated double-ended tuning fork electrometer," Sens. Actuators A Phys. 148 (2008) 395 (DOI: 10.1016/j.sna. 2008.09.010).

[19] W. Zhang and J. E.-Y. Lee: "Frequency-based magnetic field sensing using Lorentz force axial strain modulation in a double-ended tuning fork," Sens. Actuators A Phys. 211 (2014) 145 (DOI: 10.1016/j.sna.2014.01.022).

[20] C. Liu: Foundations of MEMS (Pearson Education India, 2012).

[21] T. A. Emadi and D. A. Buchanan: "Design and characterization of a capacitive micromachined transducer with a deflectable bottom electrode," IEEE Electron Device Lett. 36 (2015) 612 (DOI: 10.1109/LED.2015.2424919).

[22] K. Riaz, et al.: "Design, damping estimation and experimental characterization of decoupled 3-DoF robust MEMS gyroscope," Sens. Actuators A Phys. 172 (2011) 523 (DOI: 10.1016/j.sna.2011.09.032).

[23] J. O. Dennis, et al.: "Modelling and simulation of the effect of air damping on the frequency and quality factor of a CMOS-MEMS resonator," Appl. Math. Inf. Sci. 9 (2015) 729 (DOI: 10.12785/amis/090221). 
imaging applications," Sens. Actuators A Phys. 222 (2015) 309 (DOI: 10.1016/ j.sna.2014.11.018).

[25] I. Khan and R. Ben Mrad: "Development of a MEMS repulsive actuator for large out-of-plane force," J. Micromech. Microeng. 24 (2014) 035022 (DOI: 10.1088/0960-1317/24/3/035022).

[26] M. U. Mian, et al.: "Optical and capacitive characterization of MEMS magnetic resonator," IEICE Electron. Express 13 (2016) 20160773 (DOI: 10.1587/elex. 13.20160773).

[27] Microsensors Incorporated: MS3110 universal capacitive readout ic pin description 16-soic and die, Costa Mesa, CA (2006).

\section{Introduction}

A variety of applications use magnetic field sensing for brain function mapping, electronic compassing, target detection and magnetic anomaly sensing. Different type of detection mechanisms are employed for the magnetic field sensing such as megnetoresistive, Hall effect sensing, flux gate, in plane coils and Lorentz force. However, each mechanism has its downside and limitations mainly high offset, thermal expansion, post deposition of magnetic materials, scaling issues and power consumption $[1,2,3,4,5]$. The electronic compasses applications are dominated by magnetoresistive sensors and Hall-effect sensors. Magnetoresistive sensors require deposition of magnetic materials and Hall-effect sensors show increase sensitivity at the cost of high power consumption [6]. Various designs of magnetic sensors are using a planar spiral coil, solenoid coil or toroid coil for coupling applications such as transformers and filters [7, 8]. The coil based sensors affect volume density of the device. A three axis CMOS-MEMS based in-plane coil sensor has been reported [9], cross coupling and post micro machining for structure release are crucial in CMOS process. Lorentz force transducer is a more popular actuation mechanism for magnetic field detection. The Lorentz force depends linearly on the applied input current perpendicular to the direction of magnetic field, this force induced by a magnetic field can deform the suspended MEMS structures with small stiffness. Lorentz force sensors do not require any special magnetic materials or large area for sensing, these advantages results in small structures, having linear motion, low power consumption, and ideal for large displacement applications [10]. Multiple readout mechanisms have been employed with the Lorentz force sensors which include piezoresistive [11, 12, 13], capacitive $[3,14,15,16]$ and optical sensors which are mainly used for out-of-plane detection $[10,17]$. Resonant frequency shifts using Lorentz force has also been presented in some designs by applying axial stress resulting in increased stiffness of the sensor $[4,18,19]$. In this work we present magnetic field detection of in-plane magnetic fields detected by an out-of-plane motion of the sensor. The dynamic response due to input current causes an up and down motion at the resonant frequency of the MEMS structure due to the Lorentz force. The voltage change is then detected by a capacitance to voltage circuitry. The in-plane magnetic field detection is used for navigation and compass applications where 3 -axis $\mathrm{X}, \mathrm{Y}$, and $\mathrm{Z}$ axis magnetic field measurements are required. These magnetic fields are in orientation parallel to the 
substrate or surface that is $\mathrm{X}$ and $\mathrm{Y}$ axis hence named in-plane where $\mathrm{Z}$ axis is normally perpendicular and is called out-of-plane. The article is divided into five sections, the first section introduce the design and functioning of the sensor. The second section describes the analytical modeling of the sensor. Section 3 explains the fabrication of the sensor. Section 4 gives the simulation results of the sensor and the final section discusses the experimental setup and results obtained with the fabricated prototype.

\section{Sensor design}

Fig. 1 shows a three-dimensional Scanning Electron Microscopic (SEM) image of the Lorentz force magnetic sensor. The sensor has a center shuttle attached to four beams with equal length. The two input parallel beams close to each other a redesigned to carry the input current to the center shuttle while two beams on the side are support beams, which allow the shuttle to move only in an out-of-plane motion. Lorentz force is produced on the shuttle when an input alternating current (AC) passes through the parallel beams in the presence of an external magnetic field. The two parallel beams increase the force on the shuttle causing it to displace in an out of plane direction perpendicular to the external magnetic field. Maximum displacement of the shuttle is achieved when the input AC frequency matches the resonant frequency of the shuttle. The sensor is operated at the resonant frequency. The displacement due to the Lorentz force is detected as a capacitive change from the gap distance between the shuttle which acts as a upper movable plate with a lower stationary plate. Capacitance output from the sensor is then detected and amplified with capacitance detection circuitry. A schematic diagram of the sensor is presented in Fig. 2. Sensor parameters are given in Table I.

\section{Modeling and analysis}

The force on the shuttle linearly depends on the input current. Lorentz force is expressed as

$$
\mathrm{F}=I B l \sin (\vartheta)
$$

where $\mathrm{I}$ is the input current through the beams, B is the external magnetic field intensity, $\vartheta$ is the corresponding angle between the current and the magnetic field. Shuttle and beams are perpendicularly oriented to the magnetic field. The sinusoidal input in the beams exerts force $\mathrm{F}$ along $\mathrm{z}$ axis [3].

\subsection{Spring constant}

The shuttle of mass $m$ is suspended over the lower plate by two fix anchored beams known as support beams and other are input beams. The sensor contains four beams of equal length, width and thicknesses. The designed suspension allows the mass to oscillate easily in an out-of-plane motion while it is rigid for the other modes. Operating frequency of the sensor is matched with the resonance frequency, so that the sensor operates in model (out-of-plane). If the angle between the input current and the input beams does not correspond to right angle still the sensor is restricted to move in an out-of-plane motion due to the operating frequency. Stiffness of a single suspension is given as [20] 


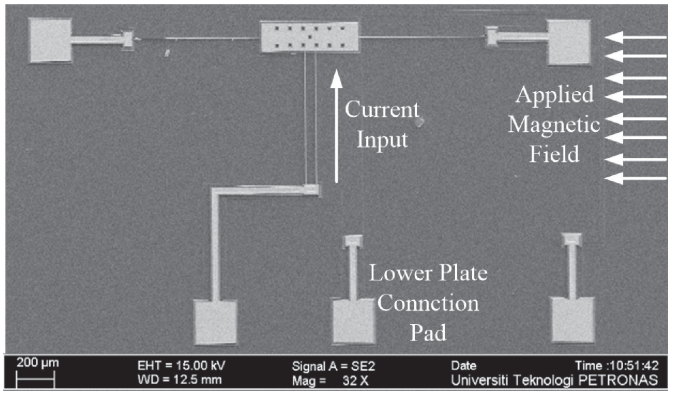

(a)

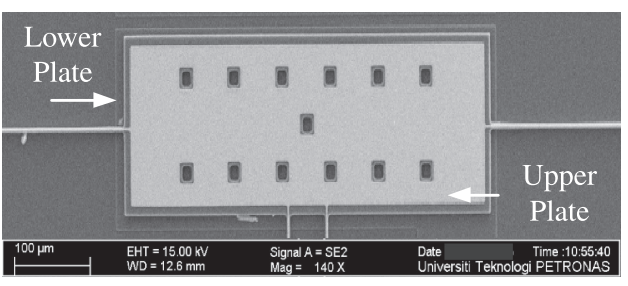

(b)

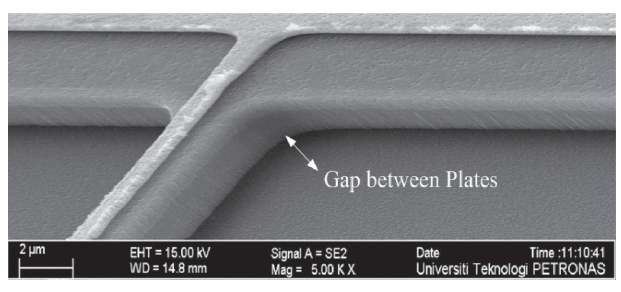

(c)

Fig. 1. (a) SEM image of the magnetic sensor, (b) shows the center shuttle with etch holes and lower capacitance plate of Poly0, (c) depicts the gap between the upper and lower plate of the sensor

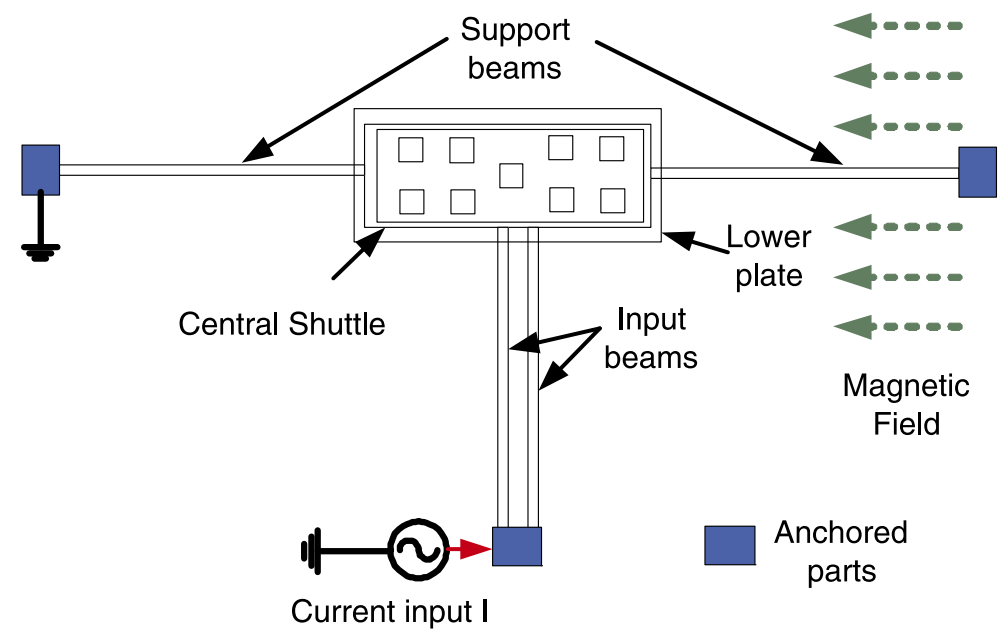

Fig. 2. Schematic of the out-of-plane sensor

$$
\mathrm{k}_{\mathrm{y}}=4\left(\frac{\mathrm{E}_{\mathrm{avg} \mathrm{W}_{\mathrm{eff}} \mathrm{t}_{\mathrm{eff}}^{3}}}{1^{3}}\right)
$$

Where $E_{\text {avg }}$ is the average Young's modulus of the material layers, $w_{\text {eff }}$ is the effective width of the beams, $t_{\text {eff }}$ is the effective thickness of the beams and 1 is the length of anchored beams.

\subsection{Spring softening effect}

When dealing with parallel plate capacitor configuration, a spring softening effect is observed. The stiffness of spring reduce due to the electrostatic force generated by the charged parallel plates. The spring softening effect is controlled by the bias voltage $[6,21]$. For out-of-plane motion, voltage dependent reduction in total stiffness is given by 
Table I. Sensor parameters

\begin{tabular}{|c|c|}
\hline Shuttle length & $322 \mu \mathrm{m}$ \\
\hline Shuttle width & $100 \mu \mathrm{m}$ \\
\hline Mass of shuttle m & $4.88 \times 10^{-10} \mathrm{~kg}$ \\
\hline Beam length 1 & $410 \mu \mathrm{m}$ \\
\hline Beam width w & $3.5 \mu \mathrm{m}$ \\
\hline Beam metal width & $1.5 \mu \mathrm{m}$ \\
\hline Effective thickness $\mathrm{t}$ & $3.5 \mu \mathrm{m}$ \\
\hline Measured gap $\mathrm{Z}_{\text {ini }}$ & $1.8 \mu \mathrm{m}$ \\
\hline Total thickness $t_{\text {total }}$ & $4 \mu \mathrm{m}$ \\
\hline
\end{tabular}

This obtained value is then subtracted from the original spring constant of the sensor. Where $8.85 \times 10^{-12} \mathrm{~F} / \mathrm{m}$ is the permittivity of free space, $\mathrm{A}$ is the total area of the capacitor plate. $z_{\text {ini }}$ is the initial distance between upper and lower plate, $V_{\text {bias }}$ is the DC biasing voltage. The spring softening effect reduces the natural frequency of the sensor depending on the input bias voltage.

\subsection{Damping coefficient}

Gases surrounding the sensor greatly effect the movement of sensor. Squeeze film damping arises when the gas between two plates with the gap $z_{\text {ini }}$ is compressed. In an out of plane motion the gas is pressed in and out of the space between plates [22]. The damping coefficient of viscosity is given as

$$
\mathrm{C}_{\mathrm{damp}}=\mu_{\mathrm{eff}}\left(\frac{A}{z_{\text {ini }}}\right)
$$

where $\mathrm{A}$ is the area of plate and $\mu_{\mathrm{eff}}$ is the effective viscosity of air including gas rarefaction effects and depends highly on the pressure which is given as

$$
\mu_{\mathrm{eff}}=\frac{\mu}{1+2 \mathrm{k}_{\mathrm{n}}+0.2 \mathrm{k}_{\mathrm{n}}^{0.788 \mathrm{e}^{-\mathrm{kn} / 10}}}
$$

where $k_{n}=\lambda / z_{\text {ini }}$ is the Knudsen number, $\lambda$ is the mean free path of the molecules.

\subsection{Density}

The average density of a MEMS structure having multiple layers is [23] given as

$$
\rho_{\text {avg }}=\frac{\rho_{\text {metal }} \times \mathrm{t}_{\text {metal }}+\rho_{\text {Poly2 }} \times \mathrm{t}_{\text {Poly } 2}+\rho_{\text {Poly } 1} \times \mathrm{t}_{\text {Poly } 1}}{\mathrm{t}_{\text {total }}}
$$

where $\rho_{\text {metal }}, \rho_{\text {poly } 1}, \rho_{\text {poly2 }}$ and $t_{\text {eff }}$ are the densities and total thickness of metal and poly layers of the PolyMUMP process. The analytically calculated sensor parameters and material properties are given in Table II. 
Table II. Sensor material properties

\begin{tabular}{lc}
\hline Spring constant $\mathrm{k}$ & $0.157 \mathrm{~N} / \mathrm{m}$ \\
\hline Spring softening $\mathrm{k}_{\text {soft }}$ & $0.061 \mathrm{~N} / \mathrm{m}$ \\
\hline Calculated resonant frequency f & $5.7 \mathrm{kHz}$ \\
\hline Biasing voltage $\mathrm{V}_{\text {bias }}$ & $1.12 \mathrm{~V}_{\mathrm{DC}}$ \\
\hline Resonant frequency with spring softening $\mathrm{f}_{\text {soft }}$ & $5.42 \mathrm{kHz}$ \\
\hline Experimental resonant frequency $\mathrm{f}_{\text {exp }}$ & $5.1 \mathrm{kHz}$ \\
\hline Damping coefficient $\mathrm{C}_{\text {damp }}$ & $3.119 \times 10^{-5} \mathrm{~kg} / \mathrm{ms}$ \\
\hline Density of PolySilicon layer $\rho_{\text {poly }}$ & $2328 \mathrm{~g} / \mathrm{cm}^{3}$ \\
\hline Density of Gold layer $\rho_{\text {gold }}$ & $19300 \mathrm{~g} / \mathrm{cm}^{3}$ \\
\hline Average Density $\rho_{\text {avg }}$ & $4495 \mathrm{~g} / \mathrm{cm}^{3}$ \\
\hline
\end{tabular}

\section{Fabrication process}

The MEMS standard fabrication process, PolyMUMPS of the MEMSCAP INC was used to fabricate the sensor. The process consists of three poly structural layers poly0, poly 1 and poly 2 having a thickness of $0.5 \mu \mathrm{m}, 2.0 \mu \mathrm{m}$ and $1.5 \mu \mathrm{m}$ respectively. A top gold metal layer is deposited on the poly2 layer having $0.5 \mu \mathrm{m}$ thickness. Sacrificial layers of Phosphor-silicate glass (PSG) oxide are used to create the separations gap between poly layers. $2.0 \mu \mathrm{m}$ between poly 0 and poly 1 and $0.75 \mu \mathrm{m}$ between poly1 and poly2. The structures are released by a wet etching process, using a $49 \%$ HydroFlouric acid followed by carbon dioxide drying to avoid stiction $[24,25]$. Fig. 3(a) shows the standard PolyMUMPs process with two separate layers poly2 and poly1, Fig. 3(b) shows double thickness PolyMUMPS process where Poly2 and Poly1 layers are combined together with poly1_poly2_via layer.

The predefined layer poly1_poly2_via connects both the poly1 and poly2 layer, which is achieved by making the width of poly1_poly2_via layer slightly wider than the poly1 and poly2 layer. The result is a double thickness layer of $4.0 \mu$ m including the top gold metal layer [26]. The design rule of top metal layer enclosure over poly 2 require a minimum width of $3.0 \mu \mathrm{m}$. For slim beams, this coverage of poly 2 which is necessary for metal, has been set to $1 \mu \mathrm{m}$ after consultation of MEMSCAP
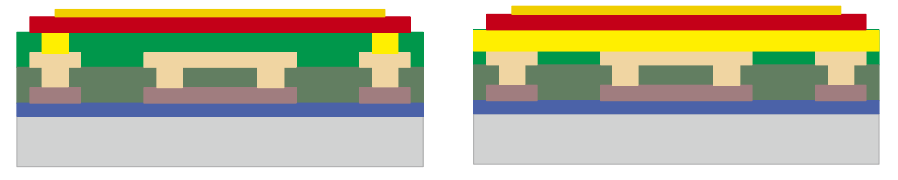

Gold

Poly2

Poly1_Poly2_via

Oxide2

Poly1

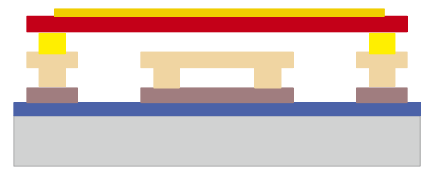

(a)

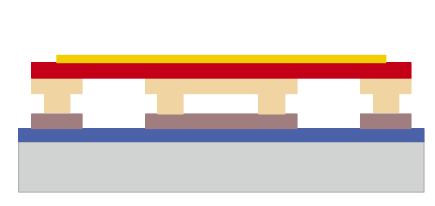

(b)

Fig. 3. (a) Standard PolyMUMP process, (b) Poly2 and Poly1 combined double thickness PolyMUMP process 


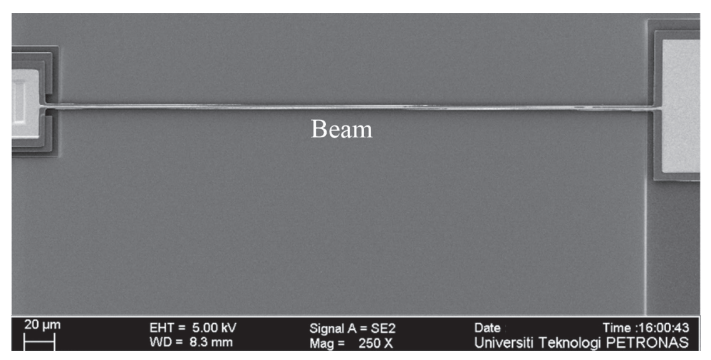

(a)

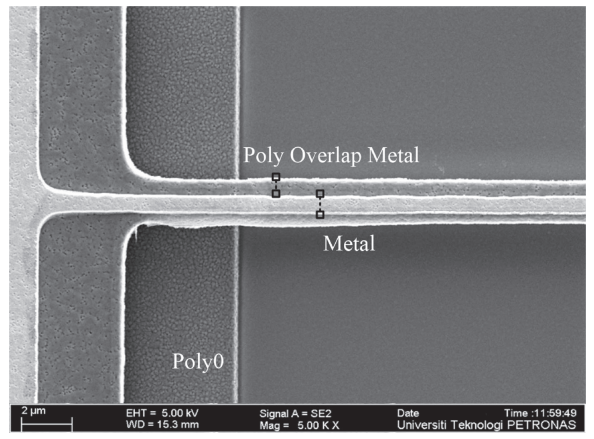

(b)

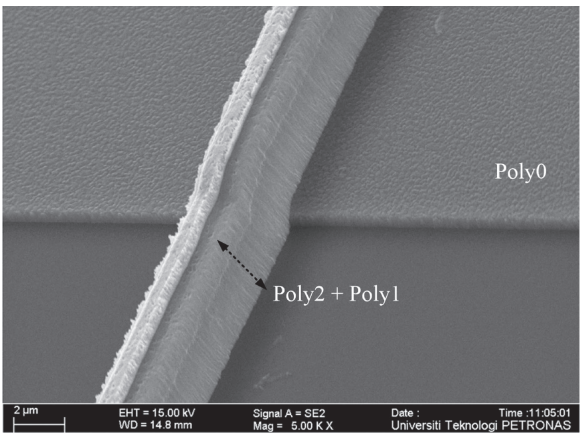

(c)
Fig. 4. (a) Fabricated support beam, (b) shows the metal layer deposited on the thin beam and Poly overlapping the metal layer, (c) side view of the double thickness support beam

INC. Fig. 4(a) depicts the fabricated double thickness beam. Fig. 4(b) shows the Poly layer overlapping the metal layer for proper release with a minimum metal designed width of $1.5 \mu \mathrm{m}$. Side view of the beam, with the top gold layer deposited over the poly2 layer is given in Fig. 4(c).

\section{Experimental results}

The sensor die is attached to a specifically designed printed circuit board (PCB) having a center area of $12 \times 12 \mathrm{~mm}$ for PolyMUMP die of size $10 \times 10 \mathrm{~mm}$. Wire bonder was used to bond the connection pads of the sensor to gold plated copper pads on the PCB using gold wires. Permanent magnets are placed perpendicular to the direction of the input current near the magnetic sensor. Permanent magnets are first measured with standard PASCO hall effect sensor prior to their use with the fabricated sensor. Fig. 5(a) shows the wire bonded die attached to the PCB along with the permanent magnet placed near the sensor. Magnetic field intensity of the permanent magnets are measured at a distance of $3.5 \mathrm{~mm}$ as this is the measured distance between the magnets and sensor over the PCB as shown in Fig. 5(b).

The capacitance detection is done by a commercially available capacitive readout circuit MS3110 of Irvine sensors. The general purpose MS3110 CMOS IC is a low noise capacitance to analog voltage converter. The MS3110 readout works either in a differential capacitor pair or a single capacitance configuration setup by setting the on board built in capacitors CS2 and CS1. The single capacitance configuration is set by setting the on board capacitors values equal to each other, the output voltage of MS3110 is constrained by the capacitance value of CS1 capacitor [27]. The maximum output voltage is limited by the capacitance 


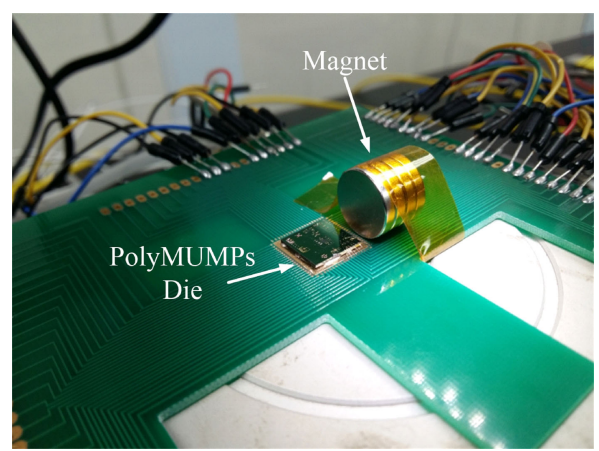

(a)

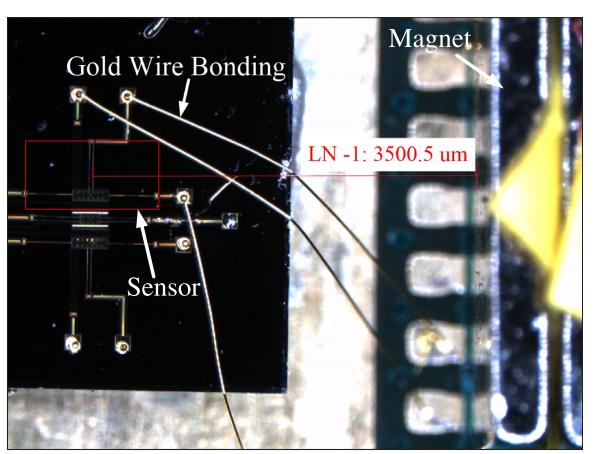

(b)

Fig. 5. (a) Magnet placed near the PolyMUMP die glued to the PCB, (b) gold wire bonding of the device pads and measured distance of the magnet to the sensor

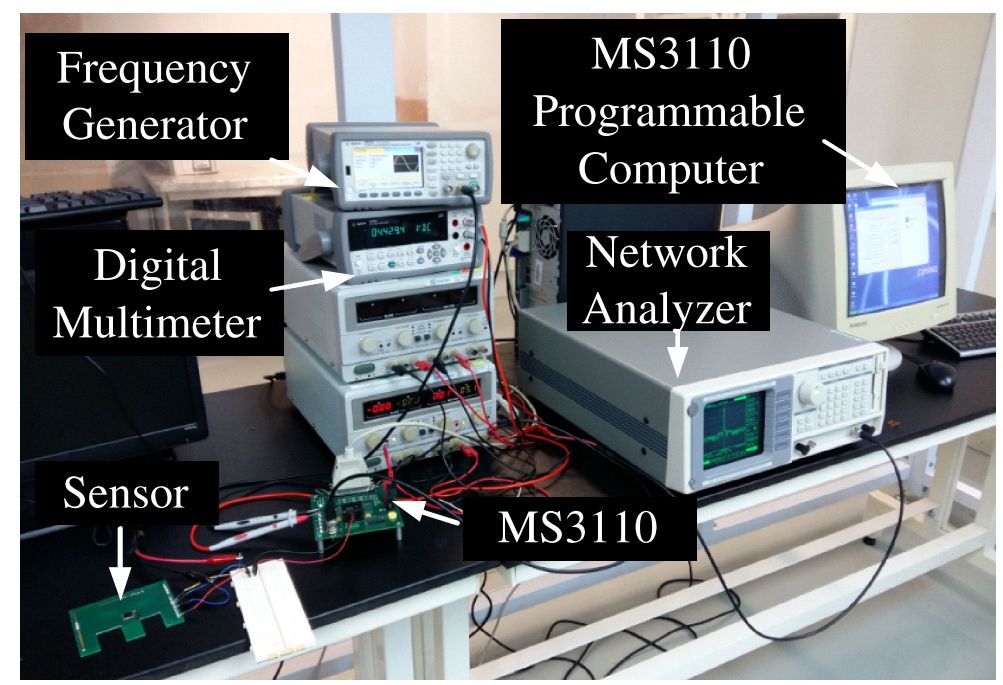

Fig. 6. Experimental setup for magnetic field measurements

value of CS1 capacitor, for this reason the highest value available for CS2 is set and made equal to the CS1 capacitor. Input to the sensor is provided by a frequency generator whereas, a constant 5 V DC supply is needed for the MS3110 circuit. The output from the capacitance to voltage readout is measured through a network analyzer. The actual experimental setup is shown in Fig. 6 .

The capacitance to voltage output of the device is measured for the input current range of $18 \mathrm{~mA}$ to $30 \mathrm{~mA}$. The maximum current limit of the sensor is $30 \mathrm{~mA}$ which limits the sensitivity of the sensor. Operating the sensor more than $30 \mathrm{~mA}$ destroys the top metal layer increasing the resistance of the sensor however the polysilicon layer does not get affected. A linear output voltage increase is measured for the magnetic flux density increment range from $11 \mathrm{mT}$ to $120 \mathrm{mT}$. Fig. 7(a) shows the linear voltage output measurement of the sensor. The increase of the sensor capacitance with the magnetic field is given in Fig. 7(b). A cyclic test for the stability of the sensor is performed. The resistance of the sensor remains constant over the period of time for multiple input current range as shown in Fig. 7(c), the slight variation of the resistance $R=32.60 \pm 0.15 \Omega$ is observed. 


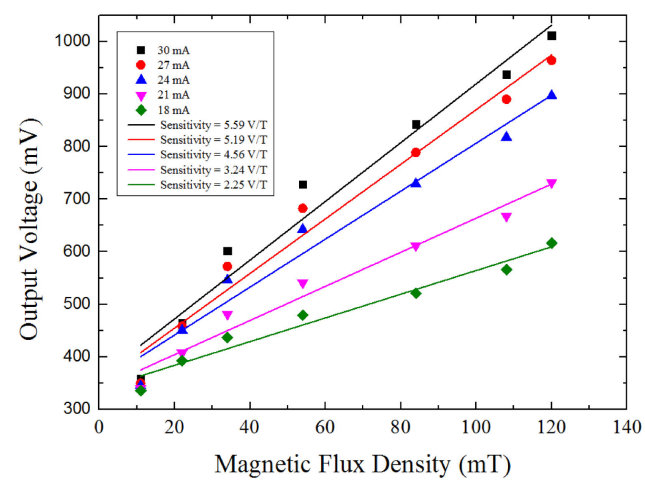

(a)

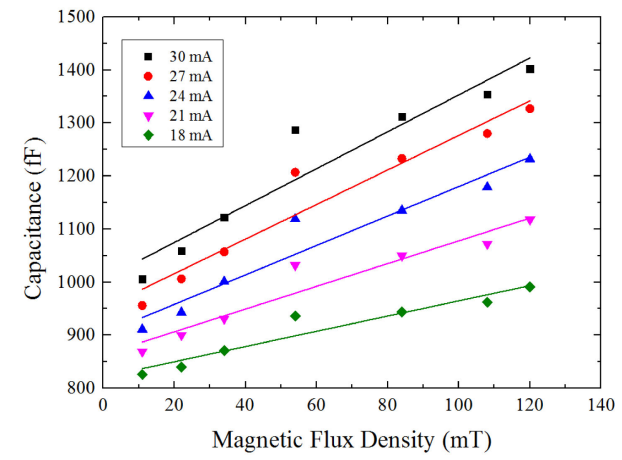

(b)

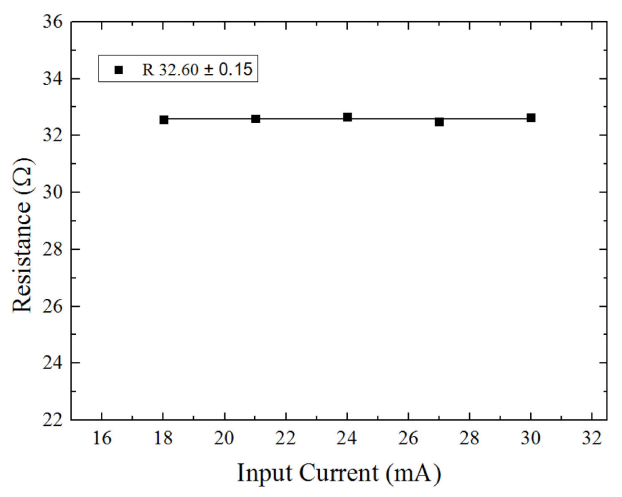

(c)

Fig. 7. (a) Measured output voltage at different magnetic field intensities, (b) capacitance change measured with the circuit, (c) variation in the resistance over the input current

The experimental extraction of the resonant frequency is conducted with a frequency generator and a capacitance detection circuit. The output is recorded by a network analyzer by varying the input frequency signals and amplitude voltage. At a magnetic field of $120 \mathrm{mT}$ and natural frequency of the sensor the highest output voltage is measured. A constant DC bias voltage $\mathrm{V}_{\text {bias }}=1.12 \mathrm{~V}_{\mathrm{DC}}$ was applied to the sensor through the pins of the MS3110 circuit. The experimentally measured resonant frequency for the out-of-plane mode fits well to the predicted analytical values considering the damping and spring softening effects which are $5.42 \mathrm{KHz}$ analytical and $5.1 \mathrm{KHz}$ experimental giving a percentage difference of $6.08 \%$ for out-of-plane motion resonant frequency. The sensitivity of the magnetic sensor input currents in the range of magnetic flux density of $11 \mathrm{mT}$ to $120 \mathrm{mT}$ are calculated in between 2.25 to $5.59 \mathrm{~V} / \mathrm{T}$.

\section{Conclusion}

In this work, a Lorentz force based PolyMUMPS MEMS magnetic field sensor is presented. The sensor was fabricated using standard MEMS fabrication process including three Poly and one metal layer. The sensor has been characterized for a range of magnetic fields using a capacitance detection circuit. The tests were performed at atmosphere pressure with varying input currents. Measurements revealed that the sensitivity increased with the input current. For the maximum current of $30 \mathrm{~mA}$ sensitivity of $5.59 \mathrm{~V} / \mathrm{T}$ was achieved. The main drawback of this 
sensor is the current limitation. The top metal layer gets destroyed when it is operated at more than $30 \mathrm{~mA}$.

\section{Acknowledgments}

Authors would like to acknowledge Ministry of Science, Technology and Information (MOSTI), Malaysia for financially supporting this research under the ScienceFund project number: 03-02-02-SF0216. 